\title{
Norm und Sanktion - Kritische Anmerkungen zum Sanktionsmodell der Norm
}

\author{
Armin Engländer"
}
A. Einführung
B. Die Begründungsargumente für das Sanktionsmodell der Norm ........... 196
C. Probleme des Sanktionsmodells der Norm 198
I. Das Problem der Normexistenz bei fehlender Sanktionierung........... 199
II. Das Problem der Normexistenz bei fehlendem Sanktionsvermeidewil- len....

\section{A. Einführung}

Die Androhung und Zufügung von Sanktionen stellt nach dem herkömmlichen normtheoretischen Verständnis ein Mittel dar, mit dem man Normen Wirksamkeit verleihen kann. Durch die Sanktionierung von Normverstößen wird ein Motiv für die Normbefolgung erzeugt und so der Einsicht Rechnung getragen, dass Normadressaten zumindest nicht ohne Weiteres bereit sind, sich stets so zu verhalten, wie es die Norm von ihnen fordert. Umstritten ist zwar, inwieweit eine solche Sanktionierung eine notwendige Bedingung für die Wirksamkeit von Normen darstellt. Manche Autoren nehmen an, Normen ohne die Stützung durch eine Sanktionspraxis könnten überhaupt keine verhaltenssteuernde Kraft erlangen. So meint schon Thomas Hobbes, ein Verbot ohne Androhung von Strafen sei vergeblich; jedes Gesetz, das eine Wirkung haben solle, müsse deshalb zweierlei enthalten: zum einen das Verbot, ein Unrecht zu tun, zum anderen die Strafandrohung für den, der es dennoch tue. ${ }^{1}$ Und in der gegenwärtigen Rechtstheorie vertritt Joseph Raz die Ansicht, ein sanktionsloses Rechtssystem sei zwar logisch denkbar, aber in menschlichen Gesellschaften empirisch nicht zu realisieren; es bedürfe hier der Flankierung durch Sanktionen, um ein Mindestmaß an Gesetzestreue sicherzustellen. ${ }^{2}$ Andere gehen dagegen zumindest von der prinzipiellen Möglichkeit aus, dass Normen auch ohne eine formelle oder informelle Sanktionspraxis wirksam werden können. Max Weber etwa sieht im Glauben an die Legitimität einer Norm - worauf auch immer dieser beruht - einen Umstand, der Normadressaten mit einer hinreichenden Häufigkeit zur Normbefol-

* Prof. Dr. Armin Engländer ist Inhaber des Lehrstuhls für Strafrecht, Strafprozessrecht, Wirtschaftsstrafrecht und Rechtsphilosophie an der Universität Passau..

1 T. Hobbes, Vom Bürger, Elemente der Philosophie III, 3. Aufl., Hamburg 1994, Kap. 14 Abschn. 7. Ebenso S. Pufendorf, Über die Pflicht des Menschen und des Bürgers nach dem Gesetz der Natur, Frankfurt a.M. 1994, Kap. 2 \$ 7.

2 J. Raz, Praktische Gründe und Normen, Frankfurt a.M. 2006, S. 216. 
gung zu veranlassen vermag. ${ }^{3}$ Und Norbert Hoerster geht in seinen Studien zur Moralphilosophie davon aus, dass die Akzeptanz einer Norm zumindest eine begrenzte handlungsmotivierende Kraft entfaltet. ${ }^{4}$ Einigkeit besteht aber ungeachtet der unterschiedlichen Einschätzungen zur Notwendigkeit einer Sanktionspraxis darüber, dass die Sanktion etwas ist, was zur Norm hinzukommt, sie aber nicht konstituiert. Die Sanktionierung begründet nicht das Verboten-Sein eines bestimmten Verhaltens, sondern setzt dieses voraus: Das Verhalten wird sanktioniert, weil es gegen eine Norm verstößt. ${ }^{5}$

Diesem Standard-Modell des Verhältnisses von Norm und Sanktion steht eine Konzeption gegenüber, die man als Sanktionsmodell der Norm bezeichnen kann. Nach dem Sanktionsmodell lässt sich, anders als die Vertreter des Standard-Modells meinen, die Sanktion nicht von der Norm abtrennen; vielmehr sind ihr zufolge Norm und Sanktion unauflöslich miteinander verbunden. Die Sanktionierung verleihe einer Norm nicht lediglich Wirksamkeit, sichere nicht bloß ihre Befolgung, sondern sie erzenge die Norm überhaupt erst. Ein Verhalten werde nicht deshalb sanktioniert, weil es verboten sei; es sei verboten, weil es sanktioniert werde. ${ }^{6}$ Anders ausgedrückt: Ohne Sanktionierung existiere keine Norm; die Sanktionspraxis bilde einen integralen Bestandteil dessen, was eine Norm ausmache. ${ }^{7}$

Ein solches sanktionstheoretisches Normverständnis liegt etwa der bekannten Befehlstheorie des Rechts des englischen Rechtstheoretikers John Austin zugrunde. ${ }^{8}$ Danach besteht das positive Recht aus allgemeinen Befehlen, die auf eine höchste politische Autorität - den Souverän - zurückgeführt werden können. Ein Befehl aber ist Austin zufolge nichts anderes als ein an andere Personen gerichteter Handlungswunsch, bei dem diesen ein Übel zugefügt wird, wenn sie ihm nicht Folge leisten. Erst der Umstand, dass im Falle der Nicht-Befolgung eine Sanktion erfolgt, macht also für Austin den Wunsch zum (Rechts-)Befehl und begründet für die Adressaten eine Gehorsamspflicht.

Sanktionstheoretische Elemente enthält auch die Reine Rechtslehre Hans Kelsens. Nach der Reinen Rechtslehre gebieten Rechtsordnungen ihren Bürgern ein bestimm-

3 M. Weber, Soziologische Grundbegriffe, $\$ 5$ Abschn. 2, in: ders., Wirtschaft und Gesellschaft, 5. Aufl., Tübingen 1972.

4 N. Hoerster, Ethik und Interesse, Stuttgart 2003, S. $50 \mathrm{ff}$.

$5 \mathrm{Zu}$ diesem traditionellen Verständnis vgl. nur H.L.A. Hart, The Concept of Law, 2. ed., Oxford: Oxford University Press 1994, S. 27; N. Hoerster, Was ist Recht?, München 2006, S. 10 ff.; M. Iorio, Regel und Grund, Berlin/New York 2011, S. 23 ff.; P. Koller, Theorie des Rechts, 2. Aufl., Wien u.a. 1997, S. 84 ff.

6 P. Stemmer, Handeln zugunsten anderer, Berlin/New York 2000, S. 101; ders., Normativität, Berlin/ New York 2008, S. 175.

$7 \mathrm{Zu}$ einer näheren Untersuchung der verschiedenen - nicht immer hinreichend klar voneinander abgegrenzten - Behauptungen einer notwendigen Verbindung von Norm und Sanktion vgl. G. Seebaß, Die sanktionistische Theorie des Sollens, in: A. Leist (Hrsg.), Moral als Vertrag?, Berlin/New York 2003, S. 155 (161 ff.).

8 J. Austin, The Province of Jurisprudence Determined, London: Weidenfeld \& Nicolson 1954, S. 9 ff. 
tes Verhalten allein dadurch, dass sie an das gegenteilige Verhalten eine Sanktion knüpfen. Folglich stelle eine Handlung oder ein Unterlassen nur deshalb ein Delikt dar, weil es mit einem Zwangsakt verknüpft sei. ${ }^{9}$ Allerdings vertritt Kelsen lediglich eine eingeschränkte Sanktionstheorie der Norm. Denn die Verbindung zwischen dem tatbestandlichen Verhalten und der Sanktion fasst er nicht als faktische Kopplung auf, sondern als normative, d.h. gesollte Zurechnung, die ihrerseits nicht durch eine Sanktion konstituiert wird. ${ }^{10}$ Das an die Rechtsorgane adressierte „Sollen“, bestimmte Verhaltensweisen zu sanktionieren, lässt sich Kelsen zufolge nicht sanktionstheoretisch explizieren. ${ }^{11}$

Seit H.L.A. Hart in seinem Werk „The Concept of Law“ Austins Befehlstheorie des Rechts einer grundlegenden und vielbeachteten Kritik unterzogen hat, ${ }^{12}$ befinden sich die Befürworter eines sanktionstheoretischen Normverständnisses auf dem Rückzug. In der aktuellen normtheoretischen Diskussion gibt es aber Bestrebungen, das Konzept einer umfassenden Sanktionstheorie der Norm zu rehabilitieren und es gegenüber dem Standard-Modell als vorzugswürdig zu erweisen. Zu nennen sind hier die Philosophen Peter Stemmer und Ernst Tugendhat sowie der Strafrechtler Andreas Hoyer. ${ }^{13}$ Insb. Stemmer hat sich um eine eingehende Rechtfertigung des sanktionstheoretischen Ansatzes bemüht. Sein Werk „Normativität“ enthält die wohl derzeit am gründlichsten ausgearbeitete und für die Vertreter des Standard-Modells herausforderndste Fassung des Sanktionsmodells der Norm.

Von Bedeutung ist die Kontroverse über das Verhältnis von Norm und Sanktion dabei nicht nur für die Normentheorie und damit für die Rechts- und Moralphilosophie. Mit ihr sind auch erhebliche rechtsdogmatische Implikationen verbunden. Träfe die sanktionstheoretische Auffassung zu, ließe sich z.B. die in der straf- und zivilrechtlichen Deliktslehre hM, dass eine Handlung, die mangels Schuld nicht sanktioniert wird, gleichwohl als Verstoß gegen ein rechtliches Verbot rechtswidrig

9 H. Kelsen, Reine Rechtslehre, 2. Aufl., Wien 1960, S. 114 ff. Entgegen der herkömmlichen normentheoretischen Klassifikation versteht Kelsen deshalb die an die Rechtsorgane gerichtete Norm, bestimmte Verhaltensweisen zu sanktionieren, als die Primärnorm und die daraus abgeleitete, an die Bürger adressierte Norm, diese strafbedrohten Verhaltensweisen zu unterlassen, als Sekundärnorm. Vgl. H. Kelsen, Allgemeine Theorie der Normen, Wien 1979, S. 43 f.

10 Kelsen, Rechtslehre (Fn. 9), S. 4 ff., S. 45 f., S. 124. Zu Kelsens Normkonzept vgl. auch H. Dreier, Rechtslehre, Staatssoziologie und Demokratietheorie bei Hans Kelsen, 2. Aufl., Baden-Baden 1990, S. 199 ff., S. 204 ff.; C. Heidemann, Die Norm als Tatsache, Baden-Baden 1997, S. 66 ff., S. 124 ff., S. $186 \mathrm{ff}$.

11 Dies übersieht J. Renzikowski, Normentheorie als Brücke zwischen Strafrechtsdogmatik und Allgemeiner Rechtslehre, ARSP 2001, S. 110 (113).

12 Vgl. Hart, Concept (Fn. 5), S. 18 ff. S. ferner S. Shapiro, Legality, Cambridge, Mass.: The Belknapp Press of Harvard University Press 2011, S. $51 \mathrm{ff}$.

13 A. Hoyer, Strafrechtsdogmatik nach Armin Kaufmann, Berlin 1997, S. 42 ff.; Stemmer, Handeln (Fn. 6), S. 39 ff., S. 73 ff.; ders., Normativität (Fn. 6); E. Tugendhat, Vorlesung über Ethik, Frankfurt a.M. 1993, S. $39 \mathrm{ff}$. 
sein kann, ${ }^{14}$ zumindest in dieser Form nicht aufrecht erhalten. ${ }^{15}$ Grund genug also, das Sanktionsmodell der Norm einer genaueren Analyse zu unterziehen. ${ }^{16}$

\section{B. Die Begründungsargumente für das Sanktionsmodell der Norm}

Zur Begründung des sanktionstheoretischen Normverständnisses bringen seine derzeitigen Befürworter im Wesentlichen zwei Argumente vor. Sie sollen hier als Überflüssigkeitsargument und als Überlegenheitsargument bezeichnet werden. Nach dem Überflüssigkeitsargument ist die Annahme sanktionsunabhängiger Normen, die durch Sanktionen lediglich motivational gestützt werden, schlicht entbehrlich. Und dem Überlegenheitsargument zufolge ermöglicht das Sanktionsmodell der Norm eine schlüssigere Erklärung der Existenz und Funktionsweise von Normen. Das gelte gerade vor dem Hintergrund eines naturalistischen Weltverständnisses, nach dem Normen nicht in einer ominösen eigenen normativen Welt existierten, sondern als Bestandteile der physikalischen Welt begriffen werden müssten. ${ }^{17}$ Überzeugen könne deshalb nur ein reduktionistischer Ansatz, der Normen und Normativität auf nichtnormative Elemente zurückführe. Damit wird unter ontologischen Vorzeichen die Zielsetzung des ethischen Naturalismus, normative Aussagen auf deskriptive Aussagen zu reduzieren, wieder aufgegriffen. ${ }^{18}$

Das Überflüssigkeitsargument wird von Hoyer (zumindest) im Hinblick auf Strafrechtsnormen vertreten. Er wendet sich mit ihm gegen die in der deutschen Strafrechtswissenschaft weitgehend geteilte Auffassung, dass eine Rechtsordnung zwei Arten von Normen enthält, nämlich zum einen Verhaltensnormen als an den einzel-

14 Für das Strafrecht s. nur U. Kindhäuser, Lehr- und Praxiskommentar StGB, 5. Aufl., Baden-Baden 2012, Vor $\mathbb{S} 13$ Rn. 5; H. Kudlich, in: H. Satzger/B. Schmitt/G. Widmaier (Hrsg.), StGB-Kommentar, Köln 2009, Vor $\mathbb{1 3}$ ff. Rn. 4; für das Zivilrecht H. Sprauch, in: Palandt, BGB-Kommentar, 71. Aufl., München 2012, Einf v $\mathbb{S} 823$ Rn. 2 ff jeweils m.w.N.

15 Freilich erscheint die herkömmliche Interpretation des Unterschieds zwischen Rechtswidrigkeit und Schuld auch auf der Grundlage des Standard-Modells der Norm nicht zweifelsfrei; zur Kritik und einem alternativen Deutungsvorschlag vgl. J. Renzikowski, in: H. Matt/J. Renzikowski, StGB-Kommentar, München 2013, Vor $\$ 13$ Rn. 39 f. Um eine Verteidigung der herrschenden Meinung bemüht sich L. Greco, Wider die jüngere Relativierung der Unterscheidung von Unrecht und Schuld, GA 2009, S. 636 ff. Den Versuch einer sanktionstheoretischen Rekonstruktion unternimmt Hoyer, Strafrechtsdogmatik, (Fn. 13), S. $82 \mathrm{ff}$.

16 Dabei greife ich z.T. auf Überlegungen aus A. Engländer, Grund und Grenzen der Nothilfe, Tübingen, 2008, S. 63 ff., zu Hoyer zurück. Vgl. zur Kritik der Hoyerschen Konzeption ferner U. Neumann, Besprechung von Andreas Hoyer, Strafrechtsdogmatik nach Armin Kaufmann, GA 1999, S. 443 ff.; Renzikowski, Normentheorie (Fn. 6), S. 110 ff. Mit Stemmer setzt sich M. Iorio, Normen, Regeln und praktische Gründe, ARSP 2010, S. 348 ff. auseinander. S. ferner die Kurzkritiken von M. Esfeld, T. Schmidt, M. Iorio und R. Forst mit Replik von P. Stemmer in DZPhil 2010, S. 145 ff.

17 Zu den Grundpositionen des philosophischen Naturalismus s. A. Beckermann, Naturwissenschaften und manifestes Weltbild, DZPhil 2012, S. 5 ff., sowie die Beiträge von G. Vollmer, Was ist Naturalismus?, und D. Koppelberg, Was ist Naturalismus in der gegenwärtigen Philosophie?, in: G. Keil/H. Schnädelbach (Hrsg.), Naturalismus, Frankfurt a.M. 2000, S. 46 ff., S. 68 ff.

18 Zur Analyse und Kritik der klassischen Spielarten des ethischen Naturalismus vgl. W. Frankena, Analytische Ethik, München 1972, S. 117 ff.; R.M. Hare, Die Sprache der Moral, Frankfurt a.M. 1972, S. 109 ff.; G.E. Moore, Principia Ethica, Stuttgart 1996, $\$ \mathbb{S} 24 \mathrm{ff}$. 
nen gerichtete Handlungsgebote, nach denen er sich in einer bestimmten Weise verhalten soll, und zum anderen Sanktionsnormen, die im Falle des Nicht-Befolgens der Verhaltensnorm eine Sanktion anordnen. ${ }^{19}$ Nach Hoyer entspricht es dem Gebot ontologischer Sparsamkeit, keine nutzlosen Entitäten zu postulieren. Die Existenz von eigenständigen Verhaltensgeboten sei aber für den vom Strafrecht bezweckten Schutz von Rechtsgütern nicht erforderlich. Rechtsgüterschutz könne ebenso gut direkt durch eine Verknüpfung des rechtsgüterverletzenden Verhaltens mit dem Eintritt eines Nachteils gewährleistet werden. Die intendierte Verhaltenssteuerung erfolge hier schlicht dadurch, dass sich für den einzelnen das Unterlassen des rechtsgüterverletzenden Verhaltens aufgrund der mit diesem verbundenen besonderen Kosten, d.h. der Nachteilszufügung, als vorteilhaft erweise. ${ }^{20}$ Die Annahme einer Kategorie von der Sanktionspraxis vorausliegenden Verhaltensgeboten erfülle daher keine sinnvolle Funktion und sei deshalb verzichtbar. ${ }^{21}$ Daraus zieht Hoyer die Konsequenz, dass auch Normsätze keine Soll-Sätze sind, kein Sollen ausdrücken, sondern gewöhnliche Aussagesätze darstellen, die besagen, dass es für den einzelnen notwendig ist, eine bestimmte Handlung zu unterlassen, wenn er den Eintritt eines Nachteils verhindern wolle. ${ }^{22}$

$\mathrm{Zu}$ einem vergleichbaren Ergebnis gelangt das von Stemmer entwickelte Überlegenheitsargument. Den Ausgangspunkt bildet dabei die Annahme, dass Normativität, entgegen einer weitverbreiteten Ansicht, ${ }^{23}$ nicht in einem Sollen, sondern in einem spezifischen Müssen besteht. ${ }^{24}$ Dieses spezifische Müssen entsteht nach Stemmer aus dem Zusammentreffen von zwei als solchen nicht-normativen Faktoren: (1) dem Wollen eines Menschen und (2) einem praktischen Müssen der notwendigen Bedingung hinsichtlich der Verwirklichung des Gewollten. Unter dem praktischen Müssen der notwendigen Bedingung versteht Stemmer den Umstand, dass die Vornahme einer bestimmten Handlung erforderlich ist, damit ein bestimmtes Ereignis eintritt. Ist der Eintritt des Ereignisses nun auch etwas, das jemand will, so entsteht für ihn aus dem praktischen Müssen der notwendigen Bedingung ein Druck, die für den Eintritt des Gewollten erforderliche Handlung zu vollziehen, und konstituiert damit nach

19 Vgl. J. Renzikowski, Die Unterscheidung von primären Verhaltens- und sekundären Sanktionsnormen in der analytischen Rechtstheorie, in: D. Dölling/V. Erb (Hrsg.), Festschrift für Karl Heinz Gössel zum 70. Geburtstag am 16. Oktober 2002, Heidelberg 2002, S. 3 ff. Grundlegend für die deutsche Strafrechtswissenschaft K. Binding, Die Normen und ihre Übertretung Bd. 1, 3. Aufl., Leipzig 1916.

20 Hoyer, Strafrechtsdogmatik (Fn. 13), S. 267.

21 Hoyer, Strafrechtsdogmatik (Fn. 13), S. 46 ff.

22 Hoyer, Strafrechtsdogmatik (Fn. 13), S. 48.

23 Vgl. nur R. Alexy, Theorie der Grundrechte, Baden-Baden 1985, S. 46; N. Hoerster, Recht (Fn. 5), S. 10 f., S. 36 ff.; H. Kelsen, Allgemeine Theorie der Normen, Wien 1979, S. 76 f.; P. Koller, Theorie (Fn. 5), S. 65 ff.; A. Marmor, Philosophy of Law, Princeton: Princeton University Press 2011, S. 1 ff.; G.H. v. Wright, Sein und Sollen, in: ders., Normen Werte und Handlungen, Frankfurt a,M. 1994, S. $19 \mathrm{ff}$.

24 Stemmer, Handeln (Fn. 6), S. 39 ff.; Normativität (Fn. 6), S. 45 ff., S. 284 ff. 
Stemmer ein normatives Müssen. ${ }^{25}$ An einem Beispiel: Nur wenn ich meine Wohnung spätestens um 06:30 Uhr verlasse, erreiche ich den Zug von Passau nach München um 06:44 Uhr. Will ich den Zug um 06:44 Uhr nehmen, stehe ich deshalb unter dem Handlungsdruck, mich um 06:30 Uhr aus dem Haus zu begeben. Zwar kann der Betreffende das „gemusste“ Verhalten auch unterlassen - aber nur um den Preis, dass sich das von ihm Gewollte dann nicht realisiert. Zusammengefasst: Ein normatives Müssen besteht nach Stemmer darin, dass jemand etwas tun oder unterlassen muss, weil nur so etwas, was er will, auch eintritt.

Dieses Verständnis von Normativität als einem spezifischen Müssen führt nun dazu, dass Stemmer die dem Standard-Modell zugrunde liegende Auffassung ablehnt, Normen seien Handlungsanweisungen, die den Willen eines Normgebers zum Ausdruck bringen, dass der Normadressat sich in einer bestimmten Weise verhalten solle. ${ }^{26}$ Denn der bloße Umstand, dass jemand an einen anderen eine Verhaltensaufforderung richte, begründe für diesen kein Müssen. Der Adressat stehe hier unter keinerlei Handlungsdruck; er könne die Verhaltensaufforderung, den Willen des Auffordernden, ignorieren, ohne dass das für ihn irgendeine nachteilige Konsequenz habe. Das bedeutet: Sollensgebote sind für Stemmer normativ impotent. ${ }^{27}$

Um ein normatives Müssen künstlich zu erzeugen - und genau darin sieht Stemmer die Funktion von Normen - bedürfe es der Verknüpfung des Verhaltens, das unterbunden werden solle, mit einer Sanktion. Sie sorge dafür, dass der Normadressat das entsprechende Verhalten unterlassen müsse, wenn er das Übel, die Sanktion, vermeiden wolle, und generiere damit den erforderlichen Handlungsdruck. Normen so Stemmer - bestehen daher in einem sanktionskonstituierten Müssen. ${ }^{28}$

\section{Probleme des Sanktionsmodells der Norm}

Die Überzeugungskraft des Sanktionsmodells der Norm hängt nun entscheidend davon ab, ob es seinen Anspruch, die Funktionsweise von Normen besser oder zumindest einfacher als das Standard-Modell erklären zu können, tatsächlich einzulösen vermag. Das erscheint bei näherer Betrachtung allerdings zweifelhaft. Fünf Problemfelder sollen hier genauer untersucht werden: (I) das Problem der Normexistenz bei fehlender Sanktionierung, (II) das Problem der Normexistenz bei fehlendem Sanktionsvermeidewillen, (III) das Problem der sanktionstheoretischen Konstruierbarkeit

25 Stemmer, Handeln (Fn. 6), S. 55 ff.; Normativität (Fn. 6), S. 35 ff.

26 Zu diesem Normverständnis s. nur Hoerster, Recht (Fn. 5), S. 36 ff.; Iorio, Regel (Fn. 5), S. 56 ff.; Seebaß, Sollen (Fn. 7), S. 190 ff.; G.H. v. Wright, Norm and Action, London: Routledge \& Kegan Paul 1963, S. $120 \mathrm{f}$.

27 Stemmer, Normativität (Fn. 6), S. 46 f., S. 165.

28 Stemmer, Normativität (Fn. 6), S. 157 ff. 
von Ermächtigungsnormen, (IV) das Problem der Normierung der Sanktionspraxis und (V) das Problem der Möglichkeit einer Normbindung. ${ }^{29}$

\section{Das Problem der Normexistenz bei fehlender Sanktionierung}

Nach dem Sanktionsmodell ist das Unterlassen einer Handlung allein deshalb geboten, d.h. „normativ gemusst“, weil ihre Vornahme mit einer Sanktion verknüpft ist, die der Normadressat vermeiden will. Verknüpfung ist dabei nicht in dem herkömmlichen deontischen Sinne zu verstehen, dass eine Sanktion erfolgen soll, ${ }^{30}$ sondern in dem faktischen Sinne, dass eine Sanktion erfolgen wird. Hier stellt sich die Frage, welche Auswirkung auf die Existenz einer Norm dann das Ausbleiben einer Sanktion trotz Vornahme der entsprechenden Handlung hat. Muss nicht die Sanktionstheorie die kontraintuitive Annahme treffen, dass z.B. ein Diebstahlsverbot in den Fällen nicht besteht, in denen die Tat nicht geahndet wird und der Dieb deshalb keinem „normativen Müssen“ zur Vermeidung der Sanktion unterliegt?

Stemmer gibt hierauf die Antwort, es könne zwar immer sein, dass eine Sanktionierung ausbleibe. Das wisse der Normadressat indes nicht vorher. Auch wenn nicht sicher sei, dass er bestraft werde, bestehe für ihn deshalb zumindest die Gefahr einer Bestrafung. Sich in diese Gefahr zu begeben, sei aber etwas, was der Normadressat nicht wolle. Es handele sich bei ihr um eine derivate Sanktion, die der Normadressat nur vermeiden könne, wenn er das entsprechende Verhalten unterlasse. Daher be-

29 Eine weitere, häufig vorgebrachte Kritik am Sanktionsmodell der Norm besteht in dem Einwand, nicht zwischen Sanktionen und Nachteilen ohne Sanktionscharakter unterscheiden zu können. So argumentiert etwa der Normlogiker Ota Weinberger, dass sich aus der bloßen Verknüpfung eines Verhaltens mit einem Übel nicht ableiten lässt, ob damit dem einzelnen dieses Verhalten verboten werden soll oder nicht. Dem Übel sehe man nicht an, ob es sich bei ihm um eine Sanktion oder einen sonstigen Nachteil wie eine Steuer handele, der anders als die Sanktion nicht darauf abziele, eine Handlung zu verbieten. Das Übel könne in beiden Fällen exakt dasselbe sein, nämlich dass jemand einen bestimmten Geldbetrag zu zahlen habe. Der Charakter des Übels lasse sich nur bestimmen, wenn man wisse, ob es als Reaktion auf den Verstoß gegen ein Verhaltensgebot verhängt werden solle oder nicht. Das aber bedeute, dass sich das Verhaltensgebot nicht aus der Übelszufügung ableiten lasse, sondern dieser vorausliegen müsse. Denn setze man das Verhaltensgebot nicht bereits voraus, sei nicht entscheidbar, ob das verhängte Übel eine Sanktion darstelle oder einen sonstigen Nachteil ohne Sanktionscharakter. Vgl. O. Weinberger, Der semantische, der juristische und der soziologische Normbegriff, in: W. Krawietz/J. Wróblewski (Hrsg.), Sprache, Performanz und Ontologie des Rechts, Festgabe für Kazimierz Opalek zum 75. Geburtstag, Berlin 1993, S. 435 (445). Ob diese Kritik am Sanktionsmodell durchschlägt, erscheint allerdings zweifelhaft. Zwar trifft es zu, dass man dem Übel als solchem zumindest nicht notwendigerweise ansieht, ob es sich bei ihm um eine Sanktion oder einen Nachteil ohne Sanktionscharakter handelt. Eine Unterscheidung lässt sich aber treffen, wenn man ermittelt, zu welchem Zweck der Normgeber das Übel zufügt, ob es ihm also allein um die Unterbindung einer unerwünschten Verhaltensweise geht (Sanktion) oder ob er zumindest auch die Erzielung staatlicher Einkünfte anstrebt (Steuer). Und diesen Zweck kann der Normgeber den Normadressaten durch terminologische Differenzierungen mitteilen, indem er für Sanktionen Begriffe wie „Strafe“ oder „Buße“ verwendet. Das Problem, Sanktionen von Nachteilen ohne Sanktionscharakter überhaupt zu unterscheiden, ist daher für das Sanktionsmodell womöglich lösbar.

30 In diesem Sinne etwa Kelsen, Rechtslehre (Fn. 9), S. 45 ff., S. 79 ff., S. 124; Koller, Theorie (Fn. 5), S. 84 f.; O. Weinberger, Rechtslogik, 2. Aufl., Berlin 1989, S. 264 f. 
stehe für ihn ein durch die derivate Sanktion erzeugtes „,normatives Müssen“, selbst wenn die primäre Sanktion, die tatsächliche Strafe, nicht verhängt werde. ${ }^{31}$

Dieser Lösungsvorschlag vermag die Problematik freilich nur zu einem Teil zu beheben. Er hilft nicht weiter in den Konstellationen, in denen der Täter einigermaßen sicher sein kann, nicht bestraft zu werden, so dass auch keine vermeidungsbedürftige Bestrafungsgefahr vorliegt. In einem solchen Fall müsste Stemmer aufgrund des Fehlens eines sanktionskonstituierten Müssens weiterhin zu dem kontraintuitiven Ergebnis gelangen, dass eine Norm nicht existiert. Am Beispiel: Kann der Dieb sicher davon ausgehen, nicht überführt zu werden, verstieße er gegen kein Verbot.

Einen anderen Ansatz zur Problemlösung entwickelt Hoyer. Er will auf die Unterscheidung zwischen der Wirksamkeit und der Gültigkeit einer Norm zurückgreifen. Erfolge auf ein bestimmtes Verhalten tatsächlich keine Sanktion, erweise sich die Norm insoweit zwar als unwirksam, nicht jedoch als ungültig. ${ }^{32}$ Was ist hier nun mit dem Begriff der Gültigkeit gemeint? Gültigkeit - so Hoyer - heiße nicht, dass der normierte Zusammenhang zwischen dem Verhalten und der Sanktion der Fall sei, sondern - am Beispiel der Rechtsordnung - dass es dem Recht entspräche, wenn er der Fall wäre. ${ }^{33}$ Wie ist das wiederum aufzufassen? Doch offenbar nicht anders, als dass die Sanktionszufügung aus Sicht der Rechtsordnung gesollt ist. Damit greift Hoyer unter der Hand auf eben jenen nicht-sanktionstheoretisch verstandenen Begriff des Sollens zurück, den er doch aus seiner Normtheorie zu eskamotieren versucht. Auch Hoyer gelingt es damit nicht, das Problem der Normexistenz bei fehlender Sanktionierung im Rahmen des Sanktionsmodells der Norm einer befriedigenden Lösung zuzuführen.

\section{Das Problem der Normexistenz bei fehlendem Sanktionsvermeidewillen}

Aber selbst wenn eine Sanktionierung erfolgt, unterliegt der Betroffene nicht ohne Weiteres einem „normativen Müssen“ im Sinne des Sanktionsmodells, das sanktionsauslösende Verhalten zu unterlassen. Ein solches „Müssen“ besteht nur, wenn er die Sanktion auch vermeiden will. Bewertet er die Sanktion dagegen nicht als Übel etwa im Falle des Täters, den die Aussicht auf eine Freiheitsstrafe nicht schreckt, weil er sich in der Justizvollzugsanstalt besser zurecht findet als in Freiheit - existiert nach dem Sanktionsmodell wiederum keine Norm, die ihm das sanktionsauslösende Verhalten untersagt. Stemmer räumt dies ausdrücklich ein und bemerkt dazu lediglich, solche Fälle kämen praktisch nur selten vor, da Menschen sich in der Regel nicht frei aussuchen könnten, was sie als Nachteil ansehen, und jedenfalls die Einbuße von Leben, Freiheit und sozialer Achtung von den allermeisten als unerwünscht emp-

31 Stemmer, Normativität (Fn. 6), S. 178 f.

32 Hoyer, Strafrechtsdogmatik (Fn. 13), S. 58.

33 Hoyer, Strafrechtsdogmatik (Fn. 13), S. 58. 
funden werde. ${ }^{34}$ Das ändert freilich nichts an dem unbefriedigenden Resultat, dass nach dem Sanktionsmodell Personen ohne einen Sanktionsvermeidewillen das sanktionsauslösende Verhalten nicht verboten ist, es ihnen also normativ freisteht, dieses Verhalten zu vollziehen. Am Beispiel: Dem Räuber, für den die Freiheitsstrafe kein Übel darstellt, wäre der Raub erlaubt. ${ }^{35}$

Im Hinblick auf den für das Sanktionsmodell erforderlichen Sanktionsvermeidewillen stellt sich aber noch ein weiteres Problem. Mögen auch die allermeisten Menschen Sanktionen grds. als unerwünschtes Übel ansehen, so gibt es doch häufiger Fälle, in denen aus ihrer Sicht der Nachteil der (oftmals ja keineswegs sicheren, sondern nur mehr oder weniger wahrscheinlichen) Sanktionierung überwogen wird durch den Vorteil, den sie durch die Vornahme der sanktionsauslösenden Handlung gewinnen und auch nur auf diesem Weg gewinnen können. Die Sanktionierung bzw. die Möglichkeit einer solchen stellt also insoweit den Preis dar, den zu zahlen sie bereit sind, um ein ihnen wichtigeres Ziel zu erreichen. ${ }^{36}$ Am Beispiel: Um die Strecke zwischen Wohnung und Arbeitsplatz so schnell wie möglich bewältigen zu können, findet sich der Autofahrer mit der Zahlung gelegentlicher Bußgelder für Geschwindigkeitsüberschreitungen ab. Gewichtet jemand den durch das sanktionsauslösende Verhalten erlangten Vorteil höher als den Nachteil der (möglichen) Sanktionierung, folgt daraus nach dem Sanktionsmodell, dass für ihn unter dem Strich kein normatives Müssen besteht, das entsprechende Verhalten zu unterlassen. Denn zwar will er nicht sanktioniert werden. Noch viel weniger will er aber den Vorteil verfehlen, den er nur durch Vornahme der sanktionsauslösenden Handlung erreichen kann. Deshalb wird er seinen auf die Sanktion bezogenen generellen Vermeidewillen in der konkreten Situation aufgeben.

Dass unter dem Strich kein normatives Müssen besteht, soll Stemmer zufolge für die Existenz der Norm allerdings nicht schädlich sein. Es genüge, dass die Sanktionierung ein pro-tanto-Müssen erzeuge, das der Handelnde bei der Abwägung als einen gegen die Vornahme der Handlung sprechenden Faktor zu berücksichtigen habe. ${ }^{37}$ Freilich ändert das nichts daran, dass der kategorische Charakter von Verhaltensgeboten hier verloren geht. Insbesondere rechtlichen und moralischen Normen kommt indes häufig die Funktion zu, Normadressaten gerade unabhängig von ihrer Motivlage ein bestimmtes Verhalten zu gebieten bzw. zu verbieten. Nach dem Sanktionsmodell muss der Normadressat dagegen das sanktionsauslösende Verhalten nur so lange unterlassen, wie ihm die Vermeidung der Sanktion wichtiger ist als die Verwirklichung eines Ereignisses, das er nur mit diesem Verhalten herbeizuführen vermag.

34 Stemmer, Normativität (Fn. 6), S. $177 \mathrm{f}$.

$35 \mathrm{Zu}$ einem realen - freilich durch eine Wahnvorstellung bedingten - Fall s. BGH NStZ 2011, 336.

36 Kritisch zur Angleichung von Sanktionen an Preise im Rahmen der Sanktionstheorie der Norm Seebaß, Sollen (Fn. 7), S. $173 \mathrm{ff}$.

37 Stemmer, Normativität (Fn. 6), S. 183. 
Das Sanktionsmodell der Norm führt somit nicht nur bei fehlender Sanktionierung, sondern ebenfalls bei fehlendem Sanktionsvermeidewillen zu unplausiblen Ergebnissen.

\section{Das Problem der sanktionstheoretischen Konstruierbarkeit von Ermächtigungsnormen}

Versteht man Normen als sanktionskonstituiertes Müssen, stellt sich die Frage, wie sich erpresserische Verhaltensaufforderungen - z.B. die Schutzgelderpressung durch den Mafia-Gangster - von den Verhaltensgeboten des Rechts oder der Moral - z.B. dem Steuerbefehl des Finanzbeamten - unterscheiden lassen. Ein sanktionskonstituiertes Müssen besteht für die Adressaten schließlich in beiden Fällen gleichermaßen. So wie der eine der Anordnung des Finanzbeamten folgen muss, wenn er nicht mit einer Geldbuße belegt werden möchte, muss der andere dem Diktat des MafiaGangsters gehorchen, wenn er nicht erschossen werden will.

Anknüpfend an Überlegungen von Kelsen ${ }^{38}$ und Hart ${ }^{39}$ sieht Stemmer den entscheidenden Unterschied darin, dass der Finanzbeamte zu seiner Forderung ermächtigt ist, der Mafia-Gangster dagegen nicht. ${ }^{40}$ Eingeführt wird damit eine neue normative Kategorie: die Ermächtigung. Fraglich ist nun, wie sich Ermächtigungen sanktionstheoretisch konstruieren lassen, besteht ihre Funktion doch darin, dem Ermächtigten eine normative Machtposition einzuräumen, ihn aber keineswegs zu zwingen (schon gar nicht durch die Androhung und Verhängung von Sanktionen), von dieser Macht auch Gebrauch zu machen. Damit scheinen sich Ermächtigungen auf den ersten Blick einem sanktionstheoretischen Verständnis der Norm zu entziehen.

Stemmers Lösung dieses Problems besteht darin, Ermächtigungen als unselbständige Bestandteile von sanktionskonstituierten Gebots- bzw. Verbotsnormen aufzufassen. ${ }^{41}$ Dem Ermächtigten werde seine normative Machtposition dadurch zugewiesen, dass durch eine Sanktionspraxis für einen anderen ein normatives Müssen begründet werde, das zu tun, was der Ermächtigte wolle. Diese Sanktionspraxis, darin bestehe die Besonderheit, werde nicht vom Ermächtigten etabliert; er bekomme sie vom Ermächtigenden gleichsam zur Verfügung gestellt. ${ }^{42}$ Am Beispiel: Da das Ausbleiben des vom Richter ordnungsgemäß geladenen Zeugen mit Ordnungsgeld geahndet wird, besteht für den Zeugen, wenn er dies vermeiden möchte, ein normatives Müssen, der Ladung des Richters nachzukommen, so dass dieser ermächtigt ist, das Erscheinen des Zeugen anzuordnen.

38 Kelsen, Rechtslehre (Fn. 9), S. 45 ff.

39 Hart, Concept (Fn. 5), S. 82 ff.

40 Stemmer, Handeln (Fn. 6), S. 51 ff., S. 107 ff.; Normativität (Fn. 6), S. 257 ff.

41 Für eine Rekonstruktion von Ermächtigungen als Elemente von (allerdings nicht sanktionstheoretisch verstandenen) Gebots- bzw. Verbotsnormen auch Hoerster, Recht (Fn. 5), S. 32 ff.

42 Stemmer, Normativität (Fn. 6), S. 244 ff. 
Eine solche sanktionstheoretische Rekonstruktion verfehlt allerdings eine wesentliche Dimension von Ermächtigungsnormen. ${ }^{43}$ Da sie Ermächtigungen lediglich als Bestandteile von sanktionskonstituierten Gebots- bzw. Verbotsnormen aufzufassen vermag, sieht sie als Normadressaten nicht die Ermächtigten, sondern nur die durch den Gebrauch der Ermächtigung Verpflichteten. Am Beispiel: Nach dem Sanktionsmodell richten sich die Kompetenznormen des GG nicht etwa an die Mitglieder von Bundestag, Bundesrat und Bundesregierung, sondern allein an den Bürger. Die wichtige Funktion von Ermächtigungsnormen, zunächst einmal den Ermächtigten über seine Machtposition und ihre Grenzen zu instruieren, wird damit nicht erfasst.

\section{Das Problem der Normierung der Sanktionspraxis}

Eine Verhaltenssteuerung durch ein sanktionskonstituiertes Müssen setzt das Bestehen einer funktionierenden Sanktionspraxis voraus. Um dies zu gewährleisten, benötigt auch die Sanktionierung selbst eine gewisse Regelung; die Androhung und Zufügung von Sanktionen muss ihrerseits normiert werden. Wie aber ist das nach dem Sanktionsmodell der Norm möglich? Allein und ausschließlich dadurch, dass auch das Sanktionsverhalten zum Gegenstand eines sanktionskonstituierten Müssens gemacht wird. Am Beispiel: Der Strafrichter muss nach den Prämissen der Sanktionstheorie gegen den für schuldig befundenen Angeklagten nur deshalb eine Strafe verhängen, weil er sonst seinerseits wegen Strafvereitelung im Amt und Rechtbeugung bestraft wird. Freilich wiederholt sich die Problematik dann auf einer höheren Ebene. Denn damit das Sanktionsverhalten zum Gegenstand eines sanktionskonstituierten Müssens wird, bedarf es einer weiteren Sanktionspraxis, die sich auf das Sanktionsverhalten erster Ordnung bezieht. Diese Sanktionspraxis zweiter Ordnung lässt sich indessen nach den Prämissen des Sanktionsmodells wiederum nur normieren durch ein weiteres sanktionskonstituiertes Müssen u.s.w. Das Sanktionsmodell gerät hier in einen infiniten Regress, aus dem es nur durch die Annahme einer ihrerseits nicht mehr normierten und damit letztlich wildwüchsigen Sanktionspraxis ausbrechen kann. Damit vermag es einem entscheidenden Kennzeichen moderner Rechtsstaaten - der intensiven Durchnormierung der staatlichen Zwangsausübung - nur unzureichend Rechnung zu tragen.

\section{Das Problem der Normbindung}

Zuletzt spricht gegen das Sanktionsmodell der Norm, dass ihm eine nicht überzeugende Vorstellung von der Steuerung menschlichen Verhaltens zugrunde liegt. Nach dem sanktionstheoretischen Verständnis kommt als Grund für das Unterlassen des sanktionsauslösenden Verhaltens - sofern der Handelnde nicht aus normunabhängigen Gründen schon entsprechend motiviert ist - nur die Sanktionsvermeidung in Betracht. Eine Normbefolgung aus intrinsischer Motivation, d.h. aufgrund einer 
Selbstbindung des Adressaten an die Norm, etwa weil er sie als gerecht ansieht oder den Normgeber als eine zur Normsetzung befugte Autorität anerkennt, scheidet dagegen aus.

Das wird von Stemmer freilich mit dem Argument bestritten, man dürfe den Existenzgrund der Norm und die Motivation des Normadressaten nicht verwechseln. Zwar existiere die Norm nur aufgrund der Sanktionspraxis. Gleichwohl könne ein Normadressat die Norm aber ohne weiteres auch deshalb befolgen, weil er sie für vernünftig halte, eine Disposition zur Normbefolgung ausgebildet habe oder die Normbefolgung für ihn einfach selbstverständlich sei. ${ }^{44}$ Dem ist indessen zu widersprechen. Versteht man die Norm nach dem Sanktionsmodell als sanktionskonstituiertes Müssen, kann der Normadressat ihr gegenüber die entsprechenden Einstellungen gar nicht einnehmen. Denn die Norm im sanktionstheoretischen Sinne stellt, wie gesehen, gerade kein kategorisches Verhaltensgebot dar, sondern besagt nur, dass der Normadressat das sanktionsauslösende Verhalten unterlassen muss, wenn er die Sanktion vermeiden will. Es gibt danach also für das Sanktionsmodell überhaupt keine unabhängig vom Sanktionsvermeidewillen befolgbare Norm, an die sich der Normadressat binden könnte.

Handlungstheoretisch muss das Sanktionsmodell der Norm damit von Akteuren ausgehen, die ihr Verhalten allein an situativen Kosten-Nutzen-Kalkulationen orientieren: Sie verrechnen in jeder Entscheidungssituation die Vorteile einer Rechtsgutsverletzung mit den möglichen Nachteilen einer hierdurch ausgelösten Sanktion. Auf der Grundlage eines ausschließlich so motivierten Handelns kann jedoch eine freiheitliche Rechtsordnung nicht dauerhaft existieren. Bedingung hierfür wäre nämlich, dass die Staatsorgane Gewalt tatsächlich nur in der Weise ausüben, wie es im Interesse der Bürger zum Schutz ihrer Rechtsgüter erforderlich ist, nicht hingegen zur Durchsetzung ihrer eigenen Interessen. Was aber sollte die Staatsorgane zu einem solchen Verhalten motivieren? Nach den von der Sanktionstheorie zugrunde gelegten Verhaltensannahmen, die ja nicht nur für die Bürger, sondern gleichermaßen für die Akteure von Legislative, Judikative und Exekutive gelten, käme wiederum nur die Furcht vor Sanktionen in Betracht. Für die Staatsorgane als Inhaber der Gewaltmittel bestünde jedoch gegenüber den weitgehend machtlosen Normalbürgern zu einer solchen Furcht kein Anlass. Es gäbe damit für sie auch kein hinreichendes Motiv, ihre Macht nicht im Wege kollusiven Zusammenwirkens zu Rechtsgutsverletzungen zum eigenen Vorteil zu missbrauchen. Eine Welt situativ entscheidender Nutzenmaximierer endete deshalb letztlich entweder in einer destruktiven Anarchie oder in einer ausbeuterischen Despotie. ${ }^{45}$ Die Existenz freiheitlich-rechtsstaatlicher Gesellschaften lässt sich daher soziologisch nur auf der Basis normgebundenen Verhaltens erklä-

44 Stemmer, Normativität (Fn. 6), S. 192 ff.

45 Eingehend dazu M. Baurmann, Der Markt der Tugend, Tübingen 1996, S. 220 ff. 
ren, ${ }^{46}$ das indes die Sanktionstheoretiker nicht in ihr Normmodell zu integrieren vermögen. Somit erweist sich das sanktionstheoretische Normverständnis auch aus sozialwissenschaftlicher Perspektive als nicht überzeugend.

\section{Die Kritik der Sanktionstheoretiker am Standard-Modell der Norm}

Bislang noch nicht entkräftet ist allerdings die Kritik, die Vertreter des sanktionstheoretischen Normverständnisses am Standard-Modell der Norm üben. Gegen die Auffassung, Normen seien Handlungsanweisungen, ${ }^{47}$ die den auf das Verhalten eines anderen gerichteten Willen eines Normgebers ausdrückten, wendet Stemmer, wie oben dargelegt, ein, der bloße Umstand, dass jemand an einen anderen eine Verhaltensaufforderung richte, begründe für diesen noch kein spezifisches Müssen. Der Adressat stehe dadurch unter keinerlei Handlungsdruck; er könne die Handlungsanweisung, den Willen des Anweisenden, ohne nachteilige Konsequenzen einfach ignorieren.

Freilich lässt sich zunächst bestreiten, dass mit dem Rekurs auf einen Handlungsdruck und ein spezifisches praktisches Müssen das Phänomen der Normativität zutreffend bestimmt wird. ${ }^{48}$ Es bedarf dieser Form des Reduktionismus auch nicht, um Normen als ontologisch unverdächtige Bestandteile unserer Wirklichkeit ansehen zu können. Das Standard-Modell der Norm zwingt keineswegs zu der (etwa von den Vertretern der Reinen Rechtslehre getroffenen) Annahme, Normen existierten in einem von der Welt des Seins getrennten eigenständigen „Reich des Sollens“. ${ }^{49}$ Und erst recht impliziert es keinen - ontologisch und erkenntnistheoretisch zweifelhaften - naturrechtlichen Standpunkt, der die objektive Geltung gewisser grundlegender moralischer Normen ganz unabhängig von menschlichen Setzungen behauptet. ${ }^{50}$

46 Der Erwerb einer Disposition, sich an Normen zu binden, kann dabei durchaus auch auf der Grundlage eigeninteressierten Verhaltens begründet werden. Vgl. dazu Baurmann, Markt (Fn. 45), S. 324 ff.; A. Engländer, Rechtsbegründung durch aufgeklärtes Eigeninteresse, JuS 2002, S. 535 (538 f.); D. Gauthier, Morals by Agreement, Oxford: Oxford University Press 1986, S. 157 ff. Zur Notwendigkeit einer freiwilligen Normbindung vgl. ferner M. Baurmann, Recht und intrinsische Motivation, in: C. Prittwitz u.a. (Hrsg.), Festschrift für Klaus Lüderssen zum 70. Geburtstag am 2. Mai 2002, Baden-Baden 2002, S. $17 \mathrm{ff}$.

47 Der Begriff der Handlungsanweisung ist dabei weit zu verstehen. Er umfasst nicht nur Gebote und Verbote, sondern auch Erlaubnisse und Ermächtigungen. Vgl. dazu auch Iorio, Regel (Fn. 5), S. 93 ff.

48 Zur Unterscheidung des „Sollens“ der Norm vom „Müssen“ der praktischen Notwendigkeit vgl. bereits $v$. Wright, Sollen (Fn. 23), S. $36 \mathrm{f}$.

49 Aus Sicht der Reinen Rechtslehre s. Kelsen, Rechtslehre (Fn. 9), S. 4 ff., S. 196 ff.; R. Thienel, Der Rechtsbegriff der Reinen Rechtslehre - eine Standortbestimmung, in: H. Schäffer u.a. (Hrsg.), Staat Verfassung - Verwaltung. Festschrift anlässlich des 65. Geburtstages von Prof. DDr. DDr. h.c. Friedrich Koja, Wien/New York 1998, S. 165 ff., S. 171 ff.; R. Walter, Hans Kelsens Rechtslehre, BadenBaden 1999, S. 10 f. Kritisch Hoerster, Recht (Fn. 5), S. 134 ff.; Marmor (Fn. 23), S. 20 ff.

50 Hinsichtlich der Frage, ob es solche naturrechtlich geltenden Normen gibt, verhält sich das StandardModell neutral. Zur Kritik der ontologischen und erkenntnistheoretischen Grundannahmen der verschiedenen Spielarten des Naturrechts vgl. A. Engländer, Moralische Richtigkeit als Bedingung der Rechtsgeltung?, ARSP 2004, S. 86 ff. Eine ausführliche Auseinandersetzung mit dem ethischen Kognitivismus, der die Möglichkeit einer Erkenntnis objektiver moralischer Richtigkeit oder Falschheit von Normen bejaht, enthält U. Czaniera, Gibt es moralisches Wissen?, Paderborn 2001. 
Versteht man Normen mit dem Standard-Modell als Handlungsanweisungen, so existiert eine Norm schlicht, sobald ein Normgeber ${ }^{51}$ gegenüber dem Normadressaten seinen Willen zum Ausdruck bringt, dass dieser sich in einer bestimmten Weise verhalten soll. ${ }^{52}$ Und sie bleibt so lange in der Welt, wie der Normgeber disponiert ist, sie aufrecht zu erhalten. ${ }^{53}$ Das gilt selbst dann, wenn der Normadressat die Handlungsanweisung zu ignorieren beschließt und dabei weiß, dass dies zu keinen nachteiligen Konsequenzen für ihn führt. Daran ist nichts ontologisch Geheimnisvolles oder gar Ominöses. Zwar wird im letzteren Fall das mit der Handlungsanweisung verfolgte Ziel, den Adressaten zu einem bestimmten Verhalten zu veranlassen, nicht erreicht. Die Handlungsanweisung wird also nicht verhaltenswirksam. Jedoch bringt sie das nicht zum Verschwinden; sie besteht, wenn der Normgeber an ihr festhält, gleichwohl weiter. ${ }^{54}$ Dass eine Norm existiert, besagt eben noch nichts darüber, ob bzw. inwieweit der Normadressat auch einen Grund hat, sie zu befolgen. ${ }^{55}$

Zudem erzeugt zwar die bloße Erteilung einer Handlungsanweisung (i.S. eines Geoder Verbots) noch keinen Handlungsdruck, kein spezifisches praktisches Müssen, wohl aber ihre Akzeptanz durch den Adressaten. Denn wird eine Handlungsanweisung erteilt, resultiert daraus zunächst die praktische Notwendigkeit, dass ihr Adressat sich in einer bestimmten Weise verhalten muss, um sie zu befolgen. Ist die Befolgung nun aber etwas, das der Adressat will, weil er die Handlungsanweisung akzeptiert (sie zu akzeptieren bedeutet, sich von ihr leiten lassen zu wollen), ${ }^{56}$ entsteht für ihn auch der entsprechende Druck, die von ihm geforderte Handlung zu vollziehen. Damit liegen alle Voraussetzungen vor, die nach Stemmer ein „normatives Müssen“ konstituieren. Geschaffen wird das spezifische praktische Müssen zu einem bestimmten Verhalten in diesem Fall jedoch ganz ohne Sanktion und einen auf Sanktions-

51 Normgeber kann sowohl ein Individuum als auch eine Gruppe von Menschen sein. Zum sich im letzteren Fall stellenden Problem der kollektiven Intentionalität näher J. Searle, Wie wir die soziale Welt machen, 2012, S. $75 \mathrm{ff.}$

52 Ebenso - mit Unterschieden im Detail - u.a. Baurmann, Markt (Fn. 45), S. 54 f.; Hoerster, Ethik (Fn. 4), S. 46 f.; Seebaß, Sollen (Fn. 7), S. 186 f. Über die Berechtigung, die Handlungsanweisung zu erteilen, ist damit freilich noch nichts gesagt. Jedoch bleibt auch eine nicht legitime Norm eine Norm. Zutreffend Seebaß a.a.O.

53 Zur Bedeutung von Willensdispositionen für die Normexistenz vgl. Baurmann, Markt (Fn. 45), S. 60 f.

54 Aber auch wenn man mit Iorio, Regel (Fn. 5), S. 59 ff., für die Existenz einer Handlungsanweisung forderte, dass der Adressat die Aufforderung des Normgebers auch akzeptiert, und anderenfalls nur von einem erfolglosen Versuch des Aufstellens einer Handlungsanweisung spräche, stützte das nicht Stemmers Auffassung. Denn die Akzeptanz der Handlungsanweisung kann aus freien Stücken erfolgen - etwa aufgrund der Überzeugung von der Legitimität - und setzt damit keinen sanktionskonstituierten Handlungsdruck voraus.

55 Normen als solche sind - entgegen einer verbreiteten Ansicht - keine Handlungsgründe. Eingehend dazu Iorio, Regel (Fn. 5), S. 148 ff.; a.A. etwa Raz, Gründe (Fn. 2), S. 66 ff.

56 Das heißt freilich nicht, dass in einer konkreten Handlungssituation andere, gegenläufige Motive sich nicht als stärker erweisen können und der Wille zur Normbefolgung daher nicht verhaltenswirksam wird. Näher zum Ganzen Hoerster, Ethik (Fn. 4), S. 50 ff. 
vermeidung gerichteten Willen. ${ }^{57}$ Und bezogen ist es auf eine Handlungsanweisung, die der Adressat als Norm befolgen möchte. Sanktionstheoretisch lässt sich dieser Vorgang der Erzeugung eines akzeptanzbegründeten Müssens daher nicht explizieren; vielmehr kann er nur auf der Grundlage des Standard-Modells der Norm angemessen erklärt werden. Setzt aber, wie oben ausgeführt, die Existenz freiheitlichrechtsstaatlicher Gesellschaften eine hinreichende Bereitschaft zu normgebundenem Verhalten seitens ihrer Mitglieder voraus, spielt das akzeptanzbegründete Müssen auch in der sozialen Realität eine ganz entscheidende Rolle. Eine genauere Betrachtung der Umstände, unter denen ein Handlungsdruck, ein spezifisches praktisches Müssen entstehen kann, spricht somit nicht gegen, sondern für das Standard-Modell der Norm.

\section{E. Fazit}

Das Sanktionsmodell der Norm bildet zwar eine theoretisch herausfordernde Alternative zum Standard-Modell der Norm. Den Anspruch, die Funktionsweise von Normen besser oder zumindest einfacher erklären zu können, kann es indes nicht einlösen. Die besseren Gründe sprechen deshalb dafür, am überkommenen Verständnis von Norm und Sanktion festzuhalten: Die Sanktion konstituiert nicht die Norm, sondern verleiht dieser lediglich - zusätzliche - Wirksamkeit.

57 Nun könnte Stemmer hier womöglich einwenden, ein solches „normatives Müssen“ entstehe nur, falls der Adressat einen Willen zur Befolgung der Handlungsanweisung besitze. Das aber sei eine Sache des Zufalls und könne nicht gezielt herbeigeführt oder gar erzwungen werden. Allerdings gilt das für die Sanktionsvermeidung, auf die Stemmer abstellt, ganz ebenso. Denn ohne den Willen des Adressaten, die Sanktion zu vermeiden, kommt es zu keinem „normativen Müssen“. Dass der Adressat die Sanktion vermeiden will, ist jedoch wiederum kontingent und lässt sich nicht erzwingen. 